\title{
HVDC-based Fast Frequency Support for Low Inertia Power Systems
}

DOI:

10.1049/cp.2017.0040

\section{Document Version}

Accepted author manuscript

Link to publication record in Manchester Research Explorer

\section{Citation for published version (APA):}

Huang, J., \& Preece, R. (2017). HVDC-based Fast Frequency Support for Low Inertia Power Systems. In The 13th IET international conference on AC and DC Power Transmission Institution of Engineering and Technology . https://doi.org/10.1049/cp.2017.0040

\section{Published in:}

The 13th IET international conference on AC and DC Power Transmission

\section{Citing this paper}

Please note that where the full-text provided on Manchester Research Explorer is the Author Accepted Manuscript or Proof version this may differ from the final Published version. If citing, it is advised that you check and use the publisher's definitive version.

\section{General rights}

Copyright and moral rights for the publications made accessible in the Research Explorer are retained by the authors and/or other copyright owners and it is a condition of accessing publications that users recognise and abide by the legal requirements associated with these rights.

\section{Takedown policy}

If you believe that this document breaches copyright please refer to the University of Manchester's Takedown Procedures [http://man.ac.uk/04Y6Bo] or contact uml.scholarlycommunications@manchester.ac.uk providing relevant details, so we can investigate your claim.

\section{OPEN ACCESS}




\title{
HVDC-based Fast Frequency Support for Low Inertia Power Systems
}

\author{
Jingya Huang and Robin Preece \\ School of Electrical and Electronic Engineering, The University of Manchester, M13 9PL, United Kingdom \\ E-mail: robin.preece@manchester.ac.uk
}

Keywords: Frequency containment, frequency stability, HVDC, low inertia.

\begin{abstract}
This paper analyses and compares different methodologies for supporting system frequency in low inertia power systems using HVDC interconnectors. Reduced system inertia, caused by the massive integration of converter-interfaced renewables and the displacement of conventional synchronous generation, can result in high values of rate of change of frequency (ROCOF), increasing the risk of frequency stability problems. In this paper, HVDC interconnections are analysed to determine their capability to provide support to the frequency stability between the Great British (GB) power system and the Continental Europe (CE) power system. Different frequency support control schemes (including droop control and the exchange of Frequency Control Reserves) are modelled and compared for a variety of cases. HVDC interconnector constraints including maximum power ramp rates and available capacity for frequency support have been derived by analysing historical operational data and incorporated into the analysis. Probabilistic uncertainty analysis considering variations in system inertia and the availability of interconnector capacity for frequency support has been performed. Studies are performed on a simplified frequency-based model of the future GB power system. The findings illustrate that droop control provides the best frequency support mechanism.
\end{abstract}

\section{Introduction}

When accessing the performance of a power system, frequency stability is a significant factor. Although it is a reasonable expectation that the frequency will vary around the nominal value, the system frequency should be always within a tolerable range in order to make sure that the power system and all equipment connected to it operates as expected. If, however, large load or generation disturbances occur, this temporary imbalance between electrical load and primary energy source (typically the mechanical torque from turbines) must be buffered by the kinetic energy within the rotors of synchronous machines. This causes the machines to speed up or slow down and therefore for the frequency to rise or fall. The rate of change of frequency (ROCOF) is dependent on the size of the disturbance and the total system inertia available to resist these changes in rotor speed. If system inertia is high with lots of synchronously connected rotating mass, the ROCOF will be smaller, providing more time for frequency control schemes to react [1]. Higher inertia, therefore, will typically lead to higher levels of frequency stability.

Sufficiently high inertia has been an inherent property of traditional power systems [2]. However, as the scale of renewable energy sources (RES) proliferates, this is changing. RES such as photovoltaic or wind energy are often interfaced through inverters. This decouples any mechanical system from the electrical system so these energy sources can be considered to be non-synchronous, providing no inertia. As RES displace conventional generation sources, this results in a low inertia system with less stable frequency behaviour.

There are three well defined levels of traditional frequency control: primary control, secondary control and tertiary control. Primary control acts to reduce the ROCOF to zero but results in steady state frequency error. It typically takes approximately $2 \mathrm{~s}$ to start and $10 \mathrm{~s}$ to be fully deployed. Secondary control then acts to reduce any steady state error and restore nominal frequency (typically in 30-60 s) and tertiary control involves the re-dispatch of generation sources to ensure suitable economic and security-based performance. For frequency stability, primary response is of critical concern.

The potential issue with existing hierarchical frequency control is the time taken for primary control to deploy in low inertia systems. A $2 \mathrm{~s}$ delay is not problematic if ROCOF is $0.05 \mathrm{~Hz} / \mathrm{s}$ but becomes catastrophic if ROCOF is $1 \mathrm{~Hz} / \mathrm{s}$ or greater. Thus, novel control schemes such as the use additional supporting control from devices such as high voltage direct current (HVDC) interconnections as well as the use of synthetic inertia are needed to ensure the frequency stability of power systems with low inertia. In this paper, the use of HVDC interconnections to provide fast frequency support is investigated.

There has been significant research into frequency control methods that can be applied to power systems with low inertia [3]-[7]. It has been highlighted in this body of work that novel frequency control for frequency stability is of critical importance to low inertia systems. Additional frequency regulation methods to maintain the stability have been analysed to reduce the frequency variability and ROCOF. In [3], the frequency response of a wind-based power system with low inertia was regulated using a droop controller. The work in [6] utilised two additional frequency control loops within wind turbines to improve the frequency stability. 
Much research has been completed on the specific topic of utilizing HVDC interconnectors to enhance the frequency stability in low inertia systems [8]-[13]. In [8], it has been shown that the HVDC frequency control scheme is able to provide frequency regulation and improve the frequency behaviour when a fault event occurs in an inertia-less system that consisting of wind turbines. The impact of HVDC interconnectors on the fluctuation of power system frequency has been analysed in [9]. In order to enhance the stability of system frequency in the power system with large penetration of wind generation, the work in [10] and [11] have proposed coordinated control strategies for the HVDC interconnectors.

This paper investigates suitable control modes for implementation with HVDC interconnectors based on the concept of exchanging power between two asynchronous areas when a sudden disturbance occurs in one area. The HVDC interconnection performances between synchronous areas Great British (GB) and Continental Europe (CE) are simulated considering anticipate inertia both under present conditions, as well as projection for the year 2025. Such a scheme would require suitable contracts and arrangements to be made to enable cross-border frequency support. It is assumed that such contracts exist and any details relating to this are outside the scope of this work.

The practical limitations of HVDC interconnectors, including permissible ramp rates and available capacity for frequency support, are considered to assess which schemes are most practically suitable. Real usage data of the links connected to the GB system is used to determine available capacity. Furthermore, uncertainty is considered by treating the system inertia and available spare capacity as stochastic. These results highlight that only relatively small amounts of capacity are required for frequency support to have a significant impact on frequency stability.

\section{Methodology}

This section describes how to model the power systems and the control frequency support control schemes of HVDC interconnection that have been applied in this project for investigations.

\subsection{System models}

A standard frequency response model of a power system is used as shown in (1). In (1), $M$ is the inertia constant $(=2 H)$, and $K L$ is the self-regulating damping caused by the load response to frequency changes.

$$
H(s)=\frac{\Delta f}{\Delta P}=\frac{1}{M s+K L}
$$

The fundamental task is to establish the parameters for this simplified model of the GB power system. These can be obtained from [14], where the inertia is given as $7560 \mathrm{MWs} / \mathrm{Hz}$, self-regulating effect $(K)$ is $1.5 \%$ and the low load demand $(L)$ is approximately $25 \mathrm{GW}$.

In order to model the low inertia system expected in the future, the inertia value reduced by one third in order to represent the impact of displacing conventional generation with converter interfaced energy sources.

The schematic configuration of the power system model can be represented as Fig. 1 where load disturbance are simulated with step change of $P_{\text {load }}$. Note that there is a $\Delta P$ signal which is used later to represent changes in power flow in any HVDC interconnections.

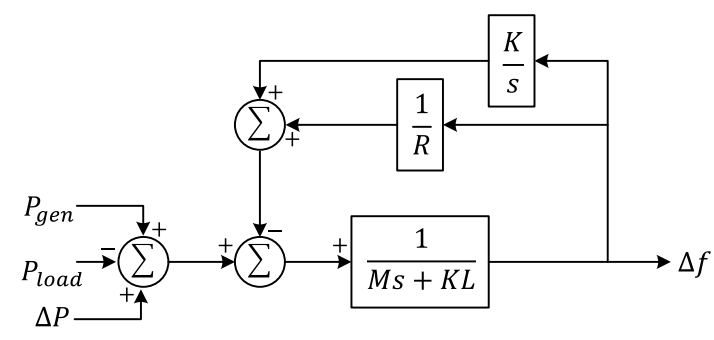

Fig. 1: Configuration of low inertia GB power system

For the CE power system, the model structure is the same but the parameters are different. The load demand of the CE power system is modelled as $320 \mathrm{GW}$, with the inertia constant being $48600 \mathrm{MWs} / \mathrm{Hz}$ and the same self-regulating effect as GB system [14].

\subsection{Droop control}

Droop control is a typical control scheme for HVDC based frequency support that has been proposed many times. It is based on standard proportional frequency control for power systems, however the HVDC system is capable of responding much faster - enabling fast frequency support through interconnectors. The controller works by determining the frequency difference between the two interconnected systems and using this as the input to a proportional transfer function that is operating with a droop gain $K_{d}$. According to [13], a reasonable value of droop gain between GB and CE power system could be $2133 \mathrm{MW} / \mathrm{Hz}$. A delay $\tau_{d}$ is also included to represent the converter delay (the time taken from requesting additional power to the converter delivering it). This is modelled as a first order delay with $\tau_{d}=0.3 \mathrm{~s}$.

All power flowing into and out of the HVDC lines must be balanced and so the use of this control following a load disturbance will in turn, disturb the second interconnected system. This will cause a frequency excursion that will need to be contained by that system's conventional frequency containment reserves, or FCR - hence the previously discussed need for appropriate contracts and agreements.

The schematic of the droop-based HVDC frequency control where the $\mathrm{GB}$ and $\mathrm{CE}$ power systems are linked by interconnectors is shown in Fig. 2 (a). Each sub-system (the GB system, and CE system) are modelled as previously outlined and shown in Fig. 1.

\subsection{Exchange of FCR control}

The other frequency support control scheme studied within this work is the concept of exchanging frequency containment reserves (FCR). This essentially means that a system will have access to the conventional FCR in the interconnected 
system via the HVDC interconnectors. Exchange of FCR is similar to droop control. However, this control scheme only uses the frequency measurement of one system (whereas the implementation of droop studied utilizes the frequency different). A delay is modelled again but now $\tau_{e}=4 \mathrm{~s}$ as this delay represents the time for the FCR in the second system to respond.

The final schematic for the exchange of FCR control scheme is shown in Fig. 2(b).

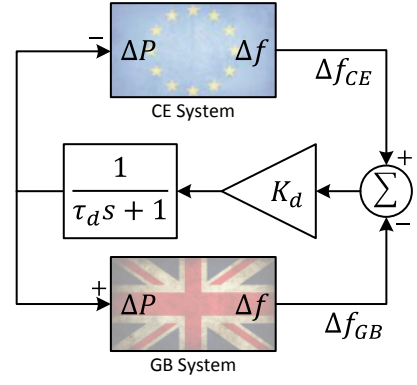

(a) Droop control

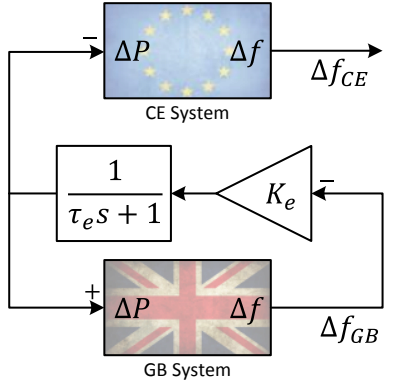

(b) Exchange of FCR
Fig. 2. HVDC-based frequency control schemes.

\subsection{Existing and future HVDC links to GB}

In order to incorporate realistic assumptions and limits of operation, it is important to establish the HVDC links that will be in operation shortly. Presently, two links exist between the GB and CE systems (IFA and BritNed) however many more are planned. The proposed HVDC links up to the year 2020 are detailed in Fig. 3. This information is used throughout the study to inform the modelling simulation parameters used.

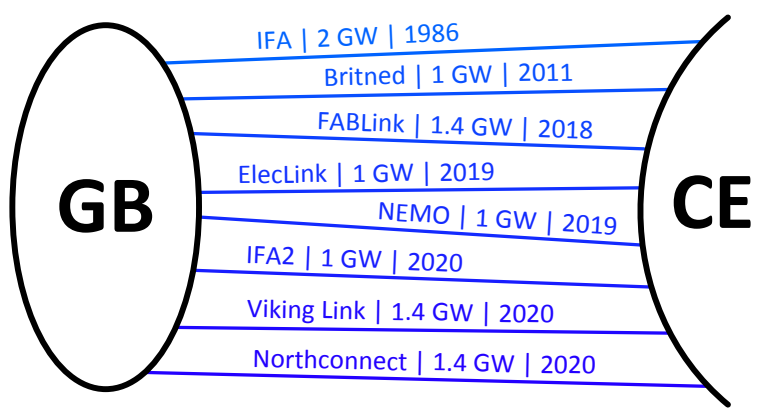

Fig. 3. Existing and future HVDC links to GB.

\section{Results and Discussions}

This section will discuss the results of the numerous studies that have been conducted. These are introduced and discussed sequentially for clarity. To improve the validity of the results, realistic system constraints including the rate of change of power in each HVDC line and total available power capacity will be taken into consideration during these studies.

\subsection{Validation of control schemes}

The first stage is to establish the frequency behaviour of the low inertia GB system model. To complete this, the GB system is subjected to a $1000 \mathrm{MW}$ load step change at a time of $10 \mathrm{~s}$. Initially, no HVDC frequency support is modelled. The frequency response of the GB system is shown in Fig. 4

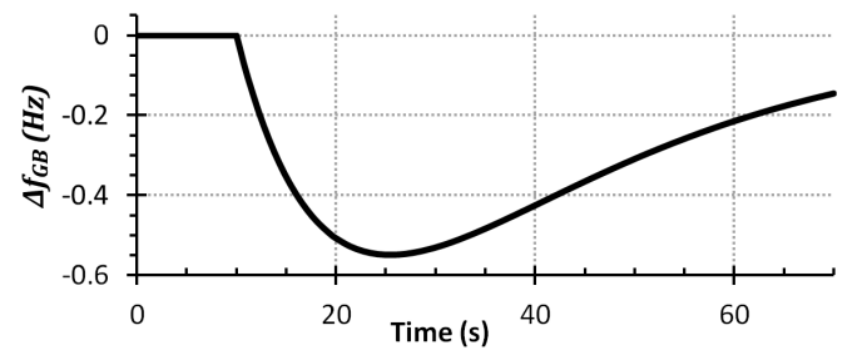

Fig. 4. GB system frequency response to load increase.

As can be seen from Fig. 4, the maximal frequency drop after the fault occurred is $0.55 \mathrm{~Hz}$. This would typically be considered unacceptable (outside $\pm 10 \%$ of nominal). To help mitigate this phenomenon, the HVDC interconnectors connected between the GB and $\mathrm{CE}$ systems are utilized for frequency support.

The rate limit for each HVDC link is assumed to be $45 \mathrm{MW} / \mathrm{s}$ as in [13]. To create a reasonable 2020 system scenario, it is assumed six HVDC links are installed and equipped for frequency support. This does not represent all the links detailed in Fig. 3.

Three case studies with different combinations of the frequency control schemes previously introduced are considered:

- Droop: all links are operating with droop control and the rate limit is set to $270 \mathrm{MW} / \mathrm{s}(=6 \times 45 \mathrm{MW} / \mathrm{s})$.

- Exchange of FCR: all links are operating with exchange of FCR control and the rate limit is set as $270 \mathrm{MW} / \mathrm{s}$.

- Droop \& Exchange of FCR: three HVDC lines operate with droop and the other three operate with exchange of FCR control. The rate limit for both schemes is set at a total of $135 \mathrm{MW} / \mathrm{s}$.

In addition, the case with no HVDC-based frequency support is also simulated (this is the same response as in Fig. 4). The frequency response of the GB power system when subjected to the same $1000 \mathrm{MW}$ disturbance as previously simulated is shown for the discussed cases in Fig. 5.

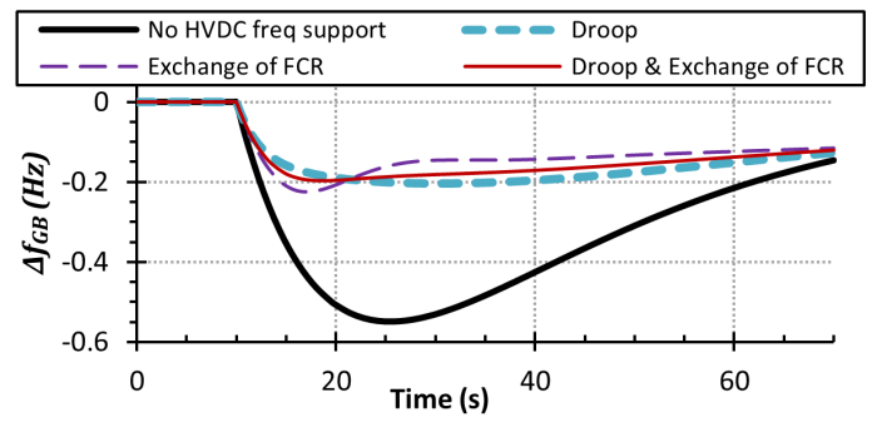

Fig. 5. GB frequency response with different control schemes.

Firstly, it is highly evident that the inclusion of frequency support from the HVDC lines makes a significant improvement to the frequency response of the system. In all cases, the frequency drop is contained to within approximately 
$0.2 \mathrm{~Hz}$ compared to over $0.5 \mathrm{~Hz}$ when the HVDC-based frequency support is not provided.

The faster response of the droop can be seen in Fig. 5 also. This faster frequency support action results in a reduced rate of change of frequency (ROCOF) compared to when exchange of FCR is used or a combination of droop and exchange of FCR is used.

\subsection{Controller gain sensitivity}

The relative performance of the droop and exchange of FCR control schemes have been investigated further by examining their sensitivity to variations in the gains settings $\left(K_{d}\right.$ and $\left.K_{e}\right)$. The impact that variations of these settings have on the maximal frequency drop in the GB system $\Delta f_{G B}^{\max }$ and the ROCOF are compared

To investigate this, the values of $K_{d}$ and $K_{e}$ are varied around their nominal values of 2133 (from 1733 to 2533 in steps of 200). The droop and exchange of FCR case studied previously described (with three lines operating with each control scheme) is used. For each controller gain combination, the system is subjected to the same $1000 \mathrm{MW}$ load disturbance in the GB system as used previously. The values of $\Delta f_{G B}^{\max }$ and ROCOF for each controller gain combination have been recorded and are shown in Table 1 and Table 2 respectively.

\begin{tabular}{|c|ccccc|}
\hline$\Delta f_{G B}^{\max }$ & \multicolumn{5}{|c|}{$K_{d}$} \\
\cline { 1 - 5 }$K_{e}$ & 1733 & 1933 & 2133 & 2333 & 2533 \\
\hline 1733 & -0.217 & -0.210 & -0.204 & -0.198 & -0.192 \\
1933 & -0.212 & -0.205 & -0.199 & -0.193 & -0.188 \\
2133 & -0.207 & -0.201 & -0.195 & -0.189 & -0.184 \\
2333 & -0.203 & -0.196 & -0.191 & -0.185 & -0.180 \\
2533 & -0.198 & -0.192 & -0.187 & -0.181 & -0.177 \\
\hline
\end{tabular}

Table 1: $\boldsymbol{\Delta} \boldsymbol{f}_{\boldsymbol{G B}}^{\max }$ values for different controller gain combinations.

\begin{tabular}{|c|ccccc|}
\hline ROCOF & \multicolumn{5}{|c|}{$K_{d}$} \\
\cline { 1 - 5 }$K_{e}$ & 1733 & 1933 & 2133 & 2333 & 2533 \\
\hline 1733 & -0.06376 & -0.06354 & -0.06332 & -0.06310 & -0.06288 \\
1933 & -0.06373 & -0.06351 & -0.06329 & -0.06307 & -0.06285 \\
2133 & -0.06371 & -0.06349 & -0.06326 & -0.06304 & -0.06283 \\
2333 & -0.06368 & -0.06346 & -0.06324 & -0.06302 & -0.06280 \\
2533 & -0.06366 & -0.06344 & -0.06321 & -0.06299 & -0.06278 \\
\hline
\end{tabular}

Table 2: GB system ROCOF values for different controller gain combinations.

It is evident from the results provided that variations in $K_{d}$ have a more significant impact on both $\Delta f_{G B}^{\max }$ and GB system ROCOF than variations in $K_{e}$. However, the differences with respect to $\Delta f_{G B}^{\max }$ are quite limited with only marginal variations between the impacts of the two controller gains. The effects on ROCOF are more significant, with variations on the droop controller having a much more noticeable effect than seen when varying $K_{e}$.

\subsection{Impact of inertia uncertainty}

To improve the robustness of the analysis, uncertainty modelling of the system inertia has been introduced to identify the variability in frequency behaviour that this causes. As inertia is dependent on the number of synchronous machines connected, it has been assumed that this is broadly correlated with the system loading which typically is modelled as following a Gaussian distribution. For this work, the inertia of the system was modelled with the previously stated value as the mean and a standard distribution $\sigma$ equal to $5 \%$ of this mean. This equates to $3 \sigma$ (or $99.7 \%$ of variability) within $\pm 15 \%$ of the mean.

This uncertainty is incorporated into the study using a Monte Carlo (repeated random sampling) approach with 1000 simulations per study. In addition, studies were performed with increasing numbers of HVDC lines as available (or not) to support system frequency. For this investigation, all HVDC lines are operating with droop control.

The results obtained for maximal frequency drop in the GB system (i.e. $\Delta f_{G B}^{\max }$ ) are shown in Fig. 6 as a boxplot. The boxplot provides a compact way of presenting the results of sampled data. The box represents the first and third quartiles ( $Q 1$ and $Q 3)$, the line within the box represents the median, and the whiskers extend to $1.5 \times(Q 3-Q 1)$ with outliers marked beyond these points.

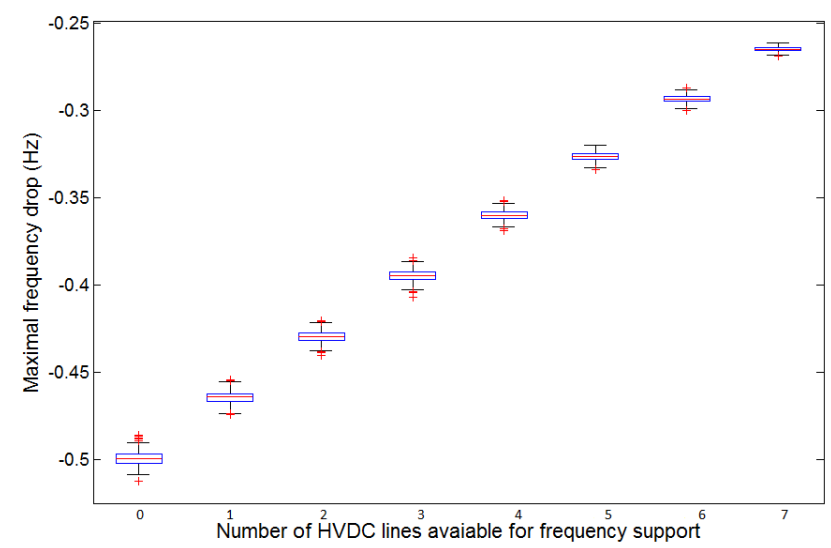

Fig. 6. Maximal frequency drops of GB system with uncertain inertia and with different numbers of available HVDC lines.

The boxes in Fig. 6 clearly show that not only does the median value of the frequency drop reduce (in absolute terms) as the number of HVDC lines supporting system frequency increases, but also the impact of the uncertainty in inertia with far less variability (i.e. box height) towards the right of the plot. Numerically, with no HVDC lines available, $\Delta f_{G B}^{\max }$ varies in the range $[-0.52,-0.48]$. Whereas with all interconnectors supporting frequency, the impact of inertia uncertainty is greatly reduced and $\Delta f_{G B}^{\max }$ varies in a much smaller range of $[-0.27,-0.26]$.

\subsection{Spare capacity analysis}

Whilst results from Section 3.3 and Fig 6 demonstrate that increasing the number of HVDC lines available for system frequency support is valuable (and helps to mitigate for uncertainty), it assumes that all lines have limitless available capacity available for frequency support. Obviously in practical power systems this would not be the case as many lines will be operating at or near their capacity limits at times and there is often little overload capacity (particularly with VSC-HVDC lines).

To include plausible and realistic limits on the capacity available for frequency support (from here onwards referred to 
simply as spare capacity), the usage data for a real HVDC interconnector between the GB and CE systems has been analysed. This has been completed by analysing the usage of BritNed over one year of operation (2015) using data extracted from [15].

The usage of the BritNed interconnector is shown in Fig. 7. A positive operating capacity means that the interconnector is importing power to the GB system from the CE system (and likewise a negative implies GB power export). It is clear that the link is often operating near maximum capacity. However, this is not always the case and some small spare capacity may be available for frequency support.

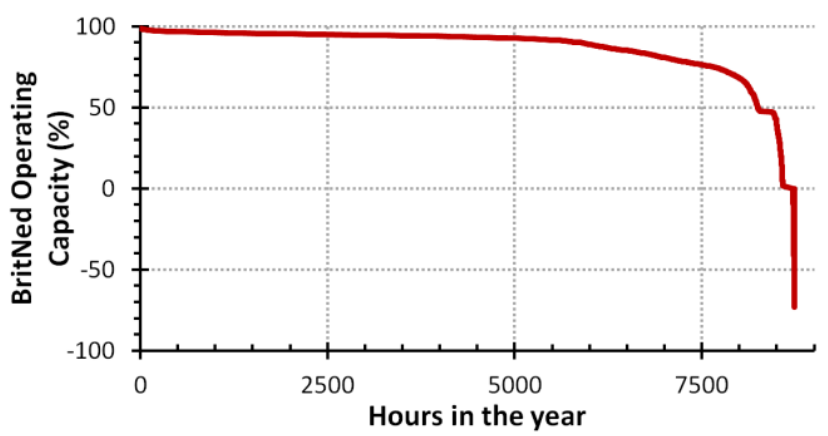

Fig. 7. BritNed operating capacity for 2015.

To model this spare capacity as an uncertain parameter, a probability density function $(p d f)$ for the spare capacity must be produced that can then be sampled during the Monte Carlo process. Spare capacity values for each of hour of the day are determined from Fig.7 simply as 100\% -operating capacity. Note that this assumes that if operating at $-100 \%$ (i.e. full capacity export from GB to $\mathrm{CE}$ ), during a low frequency event an available capacity of $200 \%$ (full power flow reversal) would be available for frequency control. However, this oversimplification is never realised as the rate limits of $45 \mathrm{MW} / \mathrm{s}$ ensure that such large power swings can never happen in the timeframes considered here.

A kernel density smoothing algorithm is used to produce the $p d f$ of spare capacity for the BritNed interconnector. This is shown as Fig. 8. This $p d f$ clearly shows that there is mostly only a small amount of spare capacity within the line available for system support. $50 \%$ of the time there is less than $7.2 \%$ spare capacity available, and $90 \%$ of the time there is less than $31.6 \%$ spare capacity. Remember it is assumed that there is zero overload capability and that the system on the other side (in this case the CE system) is able to provide this support.

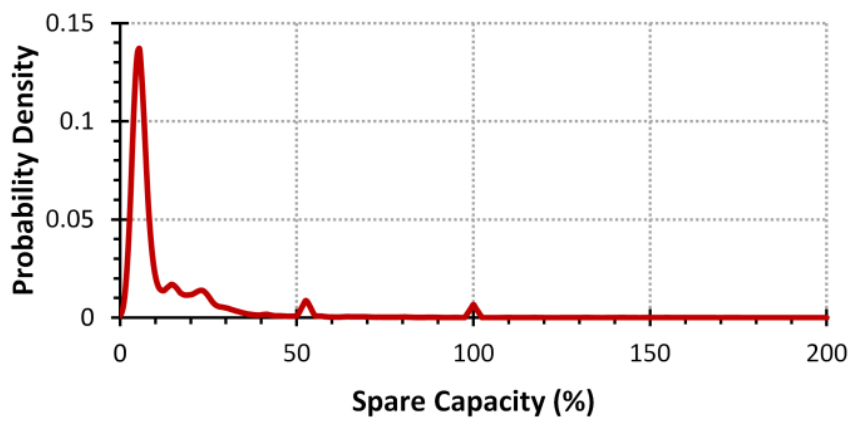

Fig. 8. Pdf of BritNed spare capacity for 2015.

\subsection{Combined uncertainty analysis}

The $p d f$ shown in Fig. 8 can be sampled from in order to include the uncertainty of the spare capacity in the analysis alongside the uncertainty of the system inertia. It is assumed that these are independent features and are not correlated. The full Monte Carlo process was performed once more modelling these aspects as uncertain. It was assumed that all links would display the same spare capacity as the BritNed link (i.e. this was sampled once per Monte Carlo run and multiplied by the total HVDC capacity). As spare capacity is likely to be based on market price differences and all links are connected between the GB and CE systems, this was deemed to be appropriate. However, the actual correlation between multiple HVDC lines between similar areas is an ongoing area of analysis.

The frequency responses obtained from all the performed simulations are shown in Fig. 9. This plot shows the range of frequency deviations that could be expected considering both the uncertainty in HVDC spare capacity and variations in inertia. This plot displays the envelope of possible responses that could be expected. Note that in all of these cases, the disturbance is the same as previously used, a $1000 \mathrm{MW}$ load increase in the GB system.

The plot in Fig. 9 shows that the maximum frequency drop in the GB system is in the range of $[-0.55,-0.28]$. This matches with the previously obtained results during this study. Fig. 9 also gives an indication of how the traces for system frequency are distributed across this envelope of performance.

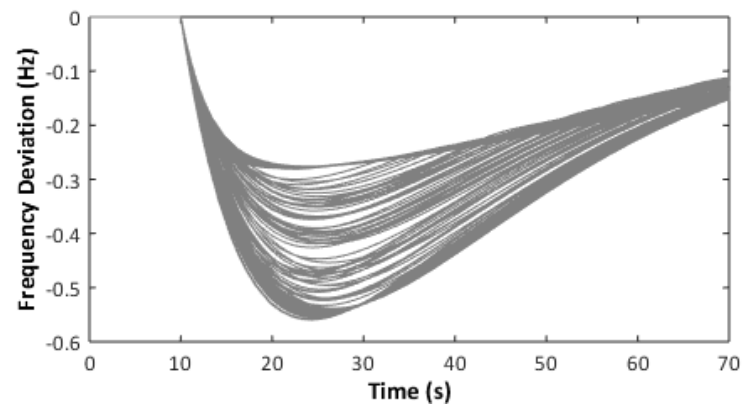

Fig. 9. GB system frequency responses considering uncertain inertia and uncertain spare capacity for frequency support.

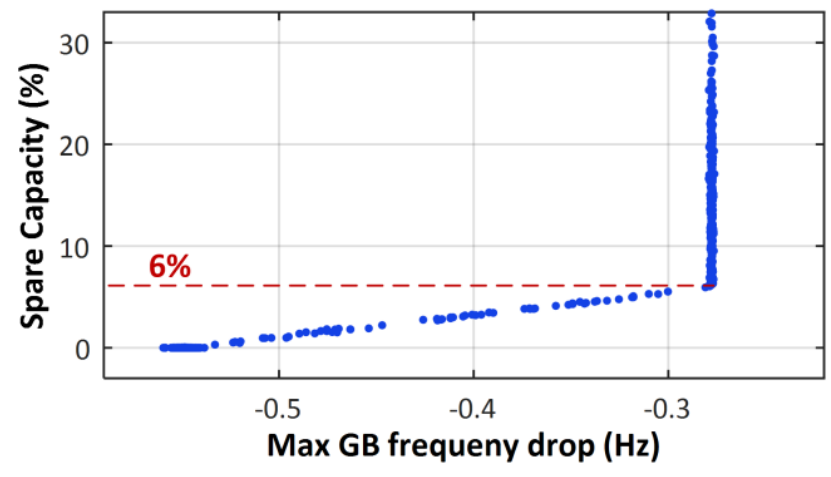

Fig. 10. Maximum GB system frequency drop against spare capacity for uncertain Monte Carlo study.

To further analyse these system responses, a plot of maximum GB system frequency drop $\left(\Delta f_{G B}^{\max }\right)$ against spare capacity 
(which was randomly sampled) has been produced and is shown as Fig. 10.

This figure shows how the availability of more spare capacity for frequency support improves the system performance and reduces $\Delta f_{G B}^{\max }$ in absolute terms. This is as expected. However, it is interesting that there's a turning point on the plot at approximately $6 \%$ spare capacity. Beyond this point, there is essentially no further gain to be made in having additional spare HVDC capacity as it cannot be deployed or used in the timeframe of interest due to the imposed rate limiters. Whilst this precise numerical result is only valid for the test conditions of this simulation- it is highly likely that a similar situation would arise in practical power systems. Furthermore, it can be seen that only a small amount of spare capacity is required in order to make sizeable improvements to the system frequency behaviour.

\section{Conclusions}

This paper has examined the ability of HVDC interconnectors to support system frequency in low inertia power systems. Different control methodologies have been examined and simplified models of the GB and CE systems have been used to explore possible control strategies for future operation.

It has been identified that droop control schemes (with their fast response) can have a more significant impact on the ROCOF in a power system experiencing a load disturbance, compared with the exchange of FCR scheme investigated. The impact of gain variations has been explored and system uncertainties have been modelled to establish the possible range of system behaviour that may be witnessed. Both system inertia and spare HVDC capacity have been modelled as uncertain. The distribution for spare capacity has been produced by analysing the usage of the BritNed interconnector using real data.

The results obtained have highlighted that with many interconnectors installed, only small amount of spare capacity are required to make significant and dramatic improvement to system frequency performance. The precise quantification of this level of spare capacity required for different systems and the value of reserving this (compared to using HVDC capacity purely for market-based power exchange) is an area of ongoing research.

\section{References}

[1] A. Ulbig, T. S. Borsche, and G. Andersson, "Impact of Low Rotational Inertia on Power System Stability and Operation," arXiv: 1312.6435v4, 2014.

[2] E. O’Neill-Carrillo, H. R. Zamot, M. Hernandez, A. A. Irizarry-Rivera, and L. O. Jimenez-Rodriguez, "Beyond traditional power systems: Energy externalities, ethics and society," in 2012 IEEE International Symposium on Sustainable Systems and Technology (ISSST), 2012, pp. $1-6$.

[3] J. M. Mauricio, A. Marano, A. Gomez-Exposito, and J. L. Martinez Ramos, "Frequency Regulation Contribution Through Variable-Speed Wind Energy
Conversion Systems," IEEE Trans. Power Syst., vol. 24, no. 1, pp. 173-180, Feb. 2009.

[4] C. He and H. Wang, "Coordination frequency control strategy design of wind farm base on time sequence control," in 2013 IEEE PES Asia-Pacific Power and Energy Engineering Conference (APPEEC), 2013, pp. 1-6.

[5] Yihui Zuo, Minxiao Han, and Zheng Chao, "Frequency stability analysis and control for thermal-wind generation with HVDC islanded sending," in TENCON 2015 - 2015 IEEE Region 10 Conference, 2015, pp. 1-6.

[6] A. Molina-Garcia, I. Munoz-Benavente, A. D. Hansen, and E. Gomez-Lazaro, "Demand-Side Contribution to Primary Frequency Control With Wind Farm Auxiliary Control," IEEE Trans. Power Syst., vol. 29, no. 5, pp. 2391-2399, Sep. 2014.

[7] W. Hu, C. Su, J. Fang, Z. Chen, and Y. Hu, “Ancillary frequency control of direct drive full-scale converter based wind power plants," in 2013 IEEE Grenoble Conference, 2013, pp. 1-6.

[8] T. M. Haileselassie, R. E. Torres-Olguin, T. K. Vrana, $\mathrm{K}$. Uhlen, and T. Undeland, "Main grid frequency support strategy for VSC-HVDC connected wind farms with variable speed wind turbines," in 2011 IEEE Trondheim PowerTech, 2011, pp. 1-6.

[9] T. Smed and G. Andersson, "Utilizing HVDC to damp power oscillations," IEEE Trans. Power Deliv., vol. 8, no. 2, pp. 620-627, Apr. 1993.

[10] H. Liu and Z. Chen, "Contribution of VSC-HVDC to Frequency Regulation of Power Systems with Offshore Wind Generation," IEEE Trans. Energy Convers., vol. 30, no. 3, pp. 918-926, 2015.

[11] Y. Wang, X. Zhu, L. Xu, and H. Li, "Contribution of VSC-HVDC connected wind farms to grid frequency regulation and power damping," in IECON 2010 - 36th Annual Conference on IEEE Industrial Electronics Society, 2010, pp. 397-402.

[12] T. Song, L. Guangkai, S. Xinli, D. Hui, and Y. Xiaohui, "A novel method for VSC-HVDC electromechanical transient modeling and simulation," in 2012 Power Engineering and Automation Conference, 2012, pp. 1-4.

[13] J. E. S. de Haan, C. Escudero Concha, M. Gibescu, J. van Putten, G. L. Doorman, and W. L. Kling, "Stabilising system frequency using HVDC between the Continental European, Nordic, and Great Britain systems," Sustain. Energy, Grids Networks, vol. 5, pp. 125-134, 2016.

[14] National Grid Electricity Transmission, "Operating the Electricity Transmission Networks in 2020," 2011.

[15] Gridwatch, "Gridwatch: UK National Gridwatch Status Jan 012015 - Dec 31 2015,” 2016. 